\title{
The effect of posterior and lateral approach on patient-reported outcome measures and physical function in patients with osteoarthritis, undergoing total hip replacement: a randomised controlled trial protocol
}

\author{
Signe Rosenlund ${ }^{1,2^{*}}$, Leif Broeng ${ }^{2}$, Carsten Jensen ${ }^{1}$, Anders Holsgaard-Larsen ${ }^{1}$ and Søren Overgaard ${ }^{1}$
}

\begin{abstract}
Background: Total hip replacement provides pain relief and improves physical function and quality of life in patients with end-stage hip osteoarthritis. The incidence of hip replacement operations is expected to increase due to the growing elderly population. Overall, the posterior approach and lateral approach are the two most commonly used approaches for hip replacement operations. The posterior approach is associated with an increased risk of revision due to dislocations, and some studies have shown that the lateral approach is associated with reduced patient-reported outcomes, including physical function and pain; however, this has not been investigated in a randomised controlled trial with a twelve-month follow-up. We hypothesized that the lateral approach has an inferior outcome in patient-reported outcome compared with the posterior approach after one year.

Methods/Design: The trial is a prospective, double blinded, parallel-group controlled trial with balanced randomisation [1: 1]. Patients with hip osteoarthritis scheduled for hip replacement surgery, aged 45-70 years, will be consecutively recruited and randomised into two groups. Group A will receive hip replacement using the posterior approach, and Group B will receive hip replacement using the lateral approach. The primary end-point for assessing the outcome of the two interventions will be twelve months after surgery. Follow-up will also be performed after three and six months. The primary outcome is Hip Disability and Osteoarthritis Outcome Score, subscale of "Physical function Short form" (HOOS-PS) Secondary outcome measures include two other subscales of HOOS ("Pain" and "Hip related Quality of Life"), physical activity level (UCLA activity score), limping (HHS) and general health status (EQ-5D-3L). Explorative outcomes include physical function test, 3D-gait-analysis and muscle strength.
\end{abstract}

Discussion: To our knowledge, this is the first randomised controlled trial comparing the posterior approach with the lateral approach with patient reported outcome as the primary outcome and with a twelve-month follow-up.

Trial registration: Clinicaltrial.gov: NCT01616667.

Keywords: Osteoarthritis, Hip replacement, Surgical approach

\footnotetext{
* Correspondence: signe.rosenlund@gmail.com

${ }^{1}$ Department of Orthopedic Surgery and Traumatology, Odense University

Hospital, Institute of Clinical Research, University of Southern Denmark,

Odense, Denmark

${ }^{2}$ Department of Orthopedic Surgery and Traumatology, Køge Hospital, Køge,

Denmark
} 


\section{Background}

Hip osteoarthritis (OA) is a common and progressive joint disease causing pain, reduced physical function and reduced quality of life. Total hip replacement (THR) provides pain reduction and improves physical function and quality of life in most patients with end-stage hip OA $[1,2]$. In the US, 427,000 hip replacements are performed each year [3]. In Denmark (DK), approximately 10,000 primary and 1,600 revision operations are performed each year, and the incidence is expected to increase due to the growing elderly population [4]. In DK, the majority (95\%) of the procedures are performed using the posterior approach (PA). In contrast, the lateral approach (LA) is more widely used internationally [5,6], and overall PA and LA are the two most commonly used approaches for THR [6,7].

PA is associated with an increased dislocation rate and revision rate due to dislocations compared with LA $[5,8,9]$. This may be due to dysfunction of the posterior soft tissue structures after PA surgery, although these are repaired in some cases [10]. Regarding patientreported outcome measures (PROM), some studies comparing PA with LA have shown that LA is associated with reduced outcome, including physical function and pain $[6,11,12]$. Furthermore pronounced limping $[13,14]$ and reduced hip abductor muscle strength may be associated with LA $[15,16]$. This might be explained by the surgical damage on the lateral structures around the hip joint $[6,7,17]$; however, these outcomes have never been evaluated in randomised controlled trials (RCT) with more than three month follow-up [18]. A Cochrane review from 2004 did not include PROMs as outcome measures due to lack of studies evaluating the patient perspective [7]. Thus, the extent to which the choice of approach affects the outcome from a patient's perspective is largely unknown [7].

To investigate the influence of surgical approach on patient-reported outcome after THR and with the perspective of reducing risk of revision, an RCT is needed that investigates potential differences between PA and LA. This trial will provide new evidence regarding the patient's perspective, including a twelve-month follow-up, upon which the choice of approach can be made.

\section{Aim}

The primary aim of this trial is to evaluate the postoperative effect of surgical approach after THR on patient-reported physical function. The secondary aim is to evaluate the effects on patient-reported pain, physical activity and quality of life; further, to evaluate objective measures of physical function, gait and hip muscle strength. We hypothesize that patient-reported and objective outcome measures within the first year will improve more in patients receiving the PA compared with LA.

\section{Methods/Design}

\section{Study design}

A prospective, double-blinded, parallel-group controlled trial with balanced randomisation [1:1], in accordance with CONSORT guidelines [19]. The trial is registered at ClinicalTrial.gov (NCT 01616667).

\section{Participants and recruitment procedure}

Patients will be referred from general medical practitioners to the outpatient clinic at the Department of Orthopaedic Surgery and Traumatology, Odense University Hospital (OUH). The clinic has two locations; one in the city of Odense at OUH, and one in the city of Svendborg at Svendborg Hospital, Denmark. Patients, aged 45-70 years, with indication for cementless THR based on symptoms, clinical and radiological findings, will be screened according to the in- and exclusion-criteria, listed in Table 1. Eligible patients will be given oral and written information about the trial. Subsequently, an appointment with the principal investigator (SR) is made, and the patients are screened again according to the exclusion criteria. The 30 second chair-stand-test (30s-CST) and Orientation-MemoryConcentration Test (OMC test) will be performed to ensure that no patients have severe medical conditions compromising their physical performance or any mentally disturbances influencing their ability to cooperate during physical testing or complete questionnaires. The patients will be given an introduction to completing a patient diary and filling in the questionnaires. Upon signing the informed consent, the baseline measures are performed. Finally, patients will be randomised and scheduled for operation by a secretary not involved in the trial.

\section{Setting}

Baseline measurement, randomisation, THR operation and follow-up will take place at OUH, Odense. The surgical intervention will be performed by a surgeon from one of two teams of experienced orthopaedic specialists. One team consists of three surgeons, all with special training in LA, and they will perform all LA operations. The other team consists of three surgeons, all with special training in PA, and they will perform all PA operations. Selection of component sizes will be based on preoperative templating performed in TraumeCad $^{\oplus}$, using standardised pelvic and anterior-posterior hip x-rays and finally adjustments on findings during surgery, if necessary. All patients will receive the same type of cementless components (Bi-metric stem ${ }^{\bullet}$ and Exceed ABT Ringloc- $\mathrm{x}$ $\left.\mathrm{Shell}^{\mathrm{TM}}\right)$. Standardised information about surgical procedure, complications, care during hospital stay and rehabilitation will be given. All patients will receive a standardised medical regime, including pre- and post- operative antibiotic within the first 24 hours, and 7 days of thromboprophylaxis. Local analgesia infiltration (150 ml Naropin ${ }^{\circ}$ 
Table 1 Criteria for participants in the trial

\begin{tabular}{|c|c|}
\hline Inclusion criteria & Exclusion criteria \\
\hline Age: $45-70$ years, both years included & More joints (hip, knee or ankle) with expected joint replacement within a year \\
\hline Patients scheduled for primary cementless total hip replacement & $\begin{array}{l}\text { Prior joint replacement on any joints (hip, knee or ankle), or any joint related } \\
\text { surgery on lower limbs, still providing symptoms }\end{array}$ \\
\hline \multirow{6}{*}{$\begin{array}{l}\text { Endstage primary hip OA or secondary OA due to mild hip } \\
\text { dysplasia (CE-angle }>20 \text { degrees) }\end{array}$} & $\mathrm{BMI}>35$ \\
\hline & Any physical disability preventing patients from walking 20 meters without aid \\
\hline & $\begin{array}{l}\text { Any neurological disease (ex. cerebral thrombosis, Parkinson) compromising the } \\
\text { walking ability }\end{array}$ \\
\hline & $\begin{array}{l}\text { Any severe medical condition compromising the physical function (ex. Chronic } \\
\text { heart failure, chronic obstructive pulmonary disease) Evaluated by 30s-CST-test }\end{array}$ \\
\hline & Severe dementia $(\mathrm{OMC}<18)$ \\
\hline & Inability to read and understand Danish writing and oral instructions \\
\hline
\end{tabular}

(2 mg/ml), $1 \mathrm{ml}$ Toradol $^{\oplus}(30 \mathrm{mg} / \mathrm{ml})$ and $0.5 \mathrm{ml}$ Adrenalin $(1 \mathrm{mg} / \mathrm{ml}))$ will be given before closure of the wound. Postoperatively, the patients will be given a standardised self-administrated analgesic regime that includes paracetamol $1000 \mathrm{mg}$ four times daily and $10 \mathrm{mg}$ morphine tablets when needed. If any additional or alternative analgesic treatment is necessary, this will be recorded in the patient dairy. The patients will be instructed on how to take the morphine tablets by the principal investigator (SR) prior to the operation and by ward nurses during the in-hospital stay. They are instructed to take a tablet if they have pain $>3$ on the numeric ranking scale (NRS) for pain (see Explorative Outcomes), when at rest in bed/chair, or when they are in pain $\geq 5$ on the NRS scale during activity. The patients will each day receive a random number (six to eight) of morphine tablets in a small plastic bag. If the patient needs more tablets, than are in the bag, the ward nurses will provide the patient with additional tablets, and this will be registered. After 24 hours, the bags will be recollected and remaining tablets will be counted and registered. The postoperative mobilisation will be performed by a physiotherapist. Standardised rehabilitation protocol (no matter which type of intervention) will be applied, including weight bearing as tolerated and no movement restrictions. The patients will be mobilised to standing position on the day of operation. The following days, the patients will be trained in transfer situations needed in daily activities (in and out of bed, up and down from chair) and walking with two canes and on stairs. Following surgery the patients are instructed in a home based rehabilitation program with 11 exercises. The 11 exercises' primary focus is strengthening of the hip muscles by use of elastic bands. The patients are instructed to conduct this program three times a day, repeating each exercise 10 times for each leg. When they can perform 30 repetitions of each exercise on the operated leg, they should increase the intensity by using an elastic band around the ankles during exercise. They are instructed to continue the exercise program for at least three months. If the physiotherapist finds it necessary, the patient will be referred to additional training provided by a local physiotherapist. The latter will be recorded.

\section{Intervention}

During the operation, all patients will be positioned in lateral decubitus position.

\section{Posterior approach}

PA is performed through an incision over the posterior part of greater trochanter through the fascia, followed by blunt dissection of gluteus maximus. Then detachment of the external rotators and incision of the posterior part of the hip capsule [20]. The hip is dislocated by internal rotation and flexion. During closure, capsular repair and re-insertion of the external rotators are performed.

\section{Lateral approach}

LA is performed through a midline incision over the greater trochanter and involves detachment of the anterior one-third of the gluteus medius insertion and gluteus minimus insertion on the tip of greater trochanter. Excision of the hip capsule is performed on the anterior side of the joint, from the basis of collum femoris to the acetabular rim. The hip is dislocated by external rotation, adduction and flexion. During closure of the wound, re-insertion of the detached part of muscle gluteus medius and muscle gluteus minimus is performed. There is no capsular repair [14]. All wounds will be closed with nylon to avoid visible suture clips on the postoperative radiographs.

\section{Outcome measures}

One primary outcome has been chosen to avoid problems with interpretations associated with multiplicity of analysis 
[19]. The primary outcome will be supported by several other patient reported secondary and tertiary outcome measures, further explored by a range of explorative outcome measures.

\section{Primary outcome \\ Physical function}

Hip Disability and Osteoarthritis Outcome Score (HOOS 2.0), subscale "Physical Function Short form (HOOS-PS)", will be used as the primary outcome, with primary endpoint after twelve months. The subscale HOOS-PS is an aggregation and shortening of the two original subscales of HOOS-ADL and Sport and Recreation. It has been developed to optimize the measurement of physical function and at the same time lower the burden of long questionnaires for the patients. The HOOS-PS subscale includes five items (three from HOOS-ADL and two from HOOS sport and recreation) that cover a wide range of physical functions, from low demand to high demand functions [21] It is a disease-specific patient-reported outcome measure developed to assess the patients' opinion about their hip associated problems [22-24]. The subscale ranges from 0 point (extreme symptoms) to 100 point (no symptoms). HOOS-PS has been evaluated regarding validity and responsiveness in THR patients. HOOS-PS was found to have high internal consistency and responsiveness [24]. HOOS 2.0 has been evaluated regarding validity and reliability in THR patients. It was found to have high content validity, construct validity, test-retest reliability, responsiveness and interpretability [22]. One study also found good internal consistency and little floor and ceiling effect [25]. All items of HOOS 2.0 has been translated into Danish [26].

\section{Secondary outcomes}

HOOS 2.0

HOOS subscale for pain, and hip related Quality of life (QoL) will also be reported.

\section{General health status}

EuroQol/EQ-5D [27] is a patient-reported generic general health questionnaire. The first part evaluates the following five dimensions; mobility, self-care, normal activities, pain/discomfort and anxiety/depression, and uses a 3-point Likert scale for each dimension. The second part evaluates the patients' perception of their overall health and is scored on a 100-point visual analogue scale. EQ-5D has been validated in knee OA patients [28], where the construct validity was found acceptable. Although EQ-5D is not validated in a THR population, it can be recommended for evaluating health related quality of life (HRQoL) with EQ-5D in these patients [29]. The test-retest reliability has been shown moderate to good in healthy and knee OA patients $[28,30]$.
The responsiveness was good in rheumatoid arthritis populations and in patients with femoral neck fractures $[31,32]$. The floor effect is negligible, but a high ceiling effect has been found in THR patients [33]. EQ-5D is translated into Danish [34].

\section{Physical activity}

The University of California Los Angeles activity score (UCLA) uses a 10-point Likert scale to evaluate activities, ranging from inactive to regular participation in impact sport or heavy labour. UCLA has been validated in patients undergoing hip or knee joint replacement [35] with good construct validity, test-retest reliability $[35,36]$, moderate responsiveness, low ceiling and floor effect [36]. UCLA contributes with important qualitative information on the patients' physical activity in correlation with other clinical outcome measures [35,37]. UCLA is translated into Danish.

\section{Limping}

Harris Hip Score (HHS) is a disease-specific surgeonreported questionnaire. It includes four domains: pain, function (subdivided in ADL and gait), deformity and joint motion. In this trial, only the question from the function domain, regarding the amount of limping, is used. It is scored on a 5-point Likert scale (none, slight, moderate, severe limp or unable to walk). The question will be used as a patient-reported outcome to evaluate the patient's own experience of limping during gait. The reliability and validity of HHS is tested in a THR population and showed low floor effect but high ceiling effect. The test-retest and inter-observer reliability was good. Internal consistency showed high Cornbach $\alpha$ value in each domain [38]. HHS is translated into Danish.

\section{Explorative outcomes \\ Physical function test}

Prior to each test, the patient will be orally instructed on how to perform the test. Regarding all maximal physical function tests, the patient is instructed to perform the test as fast as possible; however, while still feeling secure. No oral feedback will be provided during the tests. Assistive devices will be allowed at the follow-up assessments when needed, but not at baseline, according to the exclusion criteria. However, use of the arms to assist rise from the chair in 30s-CST is allowed. Both will be recorded.

\section{The $\mathbf{2 0}$ meter walk-test}

In the 20 meter walk-test (20WT), patients are instructed to walk 20 meters between two clearly visible lines marked on the floor. The time for both self-selected normal and maximal pace is measured, and the number of steps is counted. The stopwatch is started on the command "go" after a countdown from "ready, set, go". The mean 
velocity of two trials will be used for further analysis. Good agreement and excellent test-retest reliability have been demonstrated in both hip and knee OA patients [39].

\section{"Timed Up and Go" test}

The "Timed Up and Go" test (TUG) measures the time it takes a person to rise from a chair (seat height $44 \mathrm{~cm}$, with armrests), walk three meters to a clearly visible line marked on the floor, turn and walk back to the chair and sit down again. The stopwatch is started on the command "go" after a countdown from "ready, set, go". The best out of two trials will be used for further analysis [40]. TUG is used for quantifying functional mobility. Good construct validity has been reported in knee replacement patients $[41,42]$. Moderate to good test-retest and inter-rater reliability have been shown in patients with hip and knee OA [43-45].

\section{The 30 sec-chair-stand-test}

The 30 sec-Chair-Stand-test (30s-CST) measures the number of "stands" completed (seat height $44 \mathrm{~cm}$, with armrests) within 30 seconds [46]. The best out of two trials will be used for further analysis. The stopwatch is started on the command "go" after a countdown from "ready, set, go". The test assesses the overall strength of the lower limb muscles. The 30s-CST has showed valid as a measure for lower limb muscle strength in active older adults $(60+)$ [46], and the test showed good intraand inter-rater reliability in patients with moderate to end-stage hip or knee OA $[44,45,47]$.

\section{The 30 sec repeated unilateral knee bending test}

The $30 \mathrm{sec}$ repeated unilateral knee bending test (30s-knee bend) evaluates the ability to execute fast coupled eccentric-concentric muscle force, with a primary focus on knee muscle function and a secondary focus on hip muscle function. The maximum number of knee bends completed on one leg within 30 seconds will be recorded. The patient stands aligned with the front of their foot touching a straight line taped to the floor; fingertip support for balance is provided by the examiner. The patient is then asked to bend his/her knee, without forward trunk lean, until he/she is not able to see the line at their toes (about $30^{\circ}$ of knee flexion) [48]. Three to four knee bends are performed on each leg to familiarise the patient with the depth of the flexion. The unaffected leg is tested first. The stopwatch is started on the command "go" after a countdown from "ready, set, go". If the opposite (raised) leg touches the ground, the trial is stopped, and the number performed is recorded. One trial on each leg will be performed. The test was found valid to discriminate between symptomatic and non-symptomatic leg in meniscectomised patients [48]. Also, it has showed low floor and ceiling effect in meniscectomised patients [48].
It was found reliable with moderate agreement and good test-retest reliability in patients with hip and knee OA [39].

\section{Trendelenburgs test}

The Trendelenburg test will be performed according to Hardcastle et al. [49]. It indirectly measures the hip abductor muscles strength, but the validity is disputed [50,51].

\section{Hip ROM}

Passive hip range of motion (ROM) will be assessed with the patient in the supine position, and prone when assessing hip extension. Hip ROM is defined as the range of movement that an examiner is able to move the hip joint through its full range with no active participation from the patient [52]. Hip ROM in flexion/extension, abduction/adduction and internal/external rotation will be measured in degrees using a standard hand-held goniometer $(30 \mathrm{~cm})$ [53]. The reproducibility has been evaluated in patients with mild to moderate hip OA and was found poor [53].

\section{Gait analysis and hip muscle strength Gait deviation index}

Gait Deviation Index (GDI) is calculated from kinematic data collected from 3D-gait-analysis during horizontal gait. The GDI expresses the degree of gait pathology in patients compared to healthy subjects, with a mean GDI of 100 point [54]. 3D gait-analysis is extensively used to objectively collect both temporospatial, kinematic and kinetic data. GDI can simplify the complexity of the kinematic gait data in THR and other populations $[17,55,56]$. In unpublished data from our own gait laboratory, we found good test-retest reliability on GDI. GDI has been validated in children with cerebral palsy [57].

\section{Hip muscle strength}

Isometric maximal voluntary muscle contraction (MVC) will be used to assess the maximal muscle strength of the hip abduction, hip flexion and hip extension. MVC is collected standing, according to the protocol described by Jensen C. et al. [58]. MVC and rate of force development (RFD) will be analysed for both legs. A simple dice randomisation will be used to determine starting leg and the sequence of MVC exercise, avoiding a systematic leg-to-leg learning bias.

For each muscle group, three test contractions will be performed. The contraction with peak MVC will be selected for further analysis. The patient will receive both visual feedback on a monitor and verbal feedback during each test. MVC has been found reliable in THR patients [58].

\section{EMG}

Surface electromyography (sEMG) is the myoelectric signals associated with a contraction of a muscle, measured 
with a pair of surface electrodes on each muscle. We will use the SENIAM guidelines [59] for skin preparation and electrodes placement on five selected lower extremity muscles: muscle gluteus medius, muscle tensor facia lata, muscle gluteus maximus, muscle rectus femoris, and muscle semitendinosus. EMG will be collected both during gait and MVC measurement [55,60]. Peak and mean EMG signal- (absolute/normalised to MVC) and integrated EMG during stance phase will be analysed. The test-retest reliability of sEMG from muscle gluteus medius normalised to MVC has been evaluated in one study on 13 young healthy persons, and good reliability was found. Also, the intra- and inter-individual variability of EMG during gait, normalised to MVC in young healthy persons, has been found good and is therefore recommended $[61,62]$.

To ensure inter-examiner uniformity, a detailed written laboratory protocol describing the procedure and the oral instructions given to the patients during the physical function tests and MVC assessment has been developed. The protocol has been rehearsed before evaluating the study participants.

\section{Patient diary}

The patients will complete a diary consisting of four parts; 1) Pain at rest is measured four times daily using an 11-point box Numeric Ranking Scale (NRS). The NRS has been found a reliable, valid and a responsive tool in geriatric patients [63]. 2) Consumption of painkillers. Type, dosage and frequency of pain killers will be recorded. This is recommended in recent evidencebased guidelines for management of hip and knee OA [64] 3) The need for continuous cane use and 4) Completing the HOOS-pain questionnaire. The diary will be filled in each day for the first five days and then every second week until 3 months after surgery.

Digital scanning will be used for questionnaires, and manual double data entry will be done [65].

\section{Assessments and follow-up period}

Assessments point is presented in Table 2. A reminder will be sent per SMS on all the selected days for follow-up. The patients will receive the first set of questionnaires at the outpatient clinic at baseline. The following three sets, including return envelopes, will be handed out during the in-hospital stay. Furthermore, a reminder will be send per SMS at three, six and twelve months. In case of missing return, a new set and a reminder to complete the questionnaires will be sent by ordinary post service. All questionnaires will be completed at the patients' home. In case of missing visits for physical function test or assessment in the gait laboratory, the patient will be contacted by phone, and a new appointment will be scheduled.

\section{Adverse events}

A medical record audit will be performed at twelve months post-surgery. Adverse events regarding periprosthetic fracture, nerve palsy, wound infection, deep thrombosis or pulmonary embolism, dislocation and revisions will be collected. Pain will be assessed before and after all sessions of physical function test, gait-analysis and MVC measurements. Pain $>5$ on the NRS is considered an event that may influence the patients' effort of maximal performance. The assessment of pain will provide information about the feasibility of the test battery. No interim analysis or stopping guidelines are planned. Both interventions are well known and routinely performed with few severe adverse effects. However; we will register and report any adverse events to the appropriate health authorities.

\section{Sample size}

Sample size calculation was performed upon the primary outcome HOOS-PS, using one preoperative and three follow-up assessments and an estimated correlation between follow-up measurements of 0.5. We considered a minimal important difference of 10 points between the two groups at 12 months follow-up to be of clinical relevance [66-69] and used a standard deviation of 16.7 and 16.1 postoperatively [24]. We have performed our sample size calculation using the function "sampsi method(change)" supported by the Stata 13 software and described by Frison and Pocook [70]. To achieve a statistical power of $80 \%(\beta=0.80)$, it was calculated that a sample size of $n=29$ was needed in each intervention group in order to detect statistical significant differences at $\alpha=0.05$ level. $\mathrm{N}=40$ was used to account for possible drop-outs. A secondary sample size estimation was conducted to estimate the number of patients needed for analysing the primary explorative GDI. We had á prior defined that a clinically relevant improvement in gait function measured in GDI is 7.5 point, which corresponds to half a standard deviation in the only available randomised clinical trial using GDI as outcome measure [71]. From data collected in our own gait laboratory we know that the standard deviation in hip OA-patients is 9 point preoperatively [72]. To achieve a statistical power of $80 \%(\beta=0.80)$, it was calculated that a sample size of $n=17$ was needed in each intervention group in order to detect statistical significant differences at $\alpha=0.05$ level. Sample size in each group on $\mathrm{n}=20$ was used to account for possible drop-outs.

\section{Randomisation}

Balanced 1:1 block randomisation was performed using a computer-generated list containing a sequence of one letter and one number; "A" referring the patient to posterior 
Table 2 Outcomes and assessment points

\begin{tabular}{|c|c|c|c|c|c|}
\hline \multirow[b]{2}{*}{ Primary outcome } & \multirow[b]{2}{*}{ Data collection instrument (unit) } & \multicolumn{4}{|c|}{ Assessments point } \\
\hline & & Baseline & Post (days) & Post (weeks) & Post (months) \\
\hline Function of daily living & HOOS-PS & Pre & & & $3,6,12$ \\
\hline \multicolumn{6}{|l|}{ Secondary outcomes } \\
\hline \multicolumn{6}{|l|}{ Patient reported outcomes } \\
\hline Pain & HOOS-pain & Pre & & & $3,6,12$ \\
\hline Quality of life & HOOS-QoL & Pre & & & $3,6,12$ \\
\hline Physical activity & UCLA & Pre & & & $3,6,12$ \\
\hline General health & EQ-5D & Pre & & & $3,6,12$ \\
\hline Limping & Harris Hip Score & Pre & & & $3,6,12$ \\
\hline \multicolumn{6}{|l|}{ Explorative outcomes } \\
\hline \multicolumn{6}{|l|}{ Pain } \\
\hline Pain & HOOS-pain & Pre & 5 & $2-10$ & 3 \\
\hline Use of medication & (type, quantity) & Pre & $1-5$ & $2-10$ & 3 \\
\hline Short term pain measure & NRS & \multicolumn{4}{|c|}{ Before and after each test (pre- 12 months) } \\
\hline \multicolumn{6}{|l|}{ Physical function } \\
\hline 20 Meter Walk test (20WT) & Stopwatch (s and steps) & Pre & 3 & 3 & 3 \\
\hline Timed Up and Go test (TUG) & Stopwatch (s) & Pre & 3 & 3 & 3 \\
\hline Chair Stand - test (30s-CST) & (quantity) & Pre & 3 & 3 & 3 \\
\hline Knee Bend test (30s-knee bend) & (quantity) & Pre & 3 & 3 & 3 \\
\hline Trendelenburg test & (positive/negative) & Pre & & & 3 \\
\hline Joint mobility (Hip ROM) & Goniometer (degrees) & Pre & & & 3 \\
\hline \multicolumn{6}{|l|}{ Data from 3D-gait analysis } \\
\hline Total score for kinematic data & GDI (points) & Pre & & & 3,12 \\
\hline EMG & (frequency and amplitude) & Pre & & & 3,12 \\
\hline Hip Muscle strength & MVC (Nm/kg) & Pre & & & 3,12 \\
\hline Rate of forced development & 0-200 ms MVC (Nm) & Pre & & & 3,12 \\
\hline
\end{tabular}

approach, "B" referring the patient to lateral approach. " 1 " referring the patient to participate in the gait analysis and thus contributing with data on GDI, and "0" referring the patient not to participate in the gait analysis (Figure 1). Four blocks of 20 patients each was generated. The sequence was generated by a third person (JL) not involved in the trial. The letter and number combination was written on paper, folded and placed in sealed opaque consecutively numbered envelopes. The booking secretary will open the envelopes in the given order, and according to the content the patient will be scheduled for operation. In the first three blocks there will be a $66 \%$ chance of being allocated to group 1(gait analysis). This will enable us to verify the sample size calculation of $2 \times 20$ patients for the GDI. A recompilation will be made, based on ungrouped results of GDI for the 20 first actual gait analyses performed. From this, we will adjust the final number of patients allocated to gait analysis accordingly.

\section{Blinding}

The patients will be blinded to treatment. The patients are told prior to participation that they will not be informed about the type of intervention and they are carefully explained the reasons for this. Also the health care providers (doctors, nurses and physiotherapists) are all explained the importance of not talking about the intervention with the patients, and since the patients post operatively are treated after the same rehabilitations protocol, there is no need to discuss interventions. Blinding to treatment allocation (surgeons, ward nurses and, physiotherapists) is not possible due to the nature of the intervention. However, the data collectors (except one (DBN) performing gait analysis) and the principal investigator (SR) analysing the data will be blinded throughout the trial. We will use recoded identification numbers when analysing the data. The recoding will be performed by an independent person (JL). 


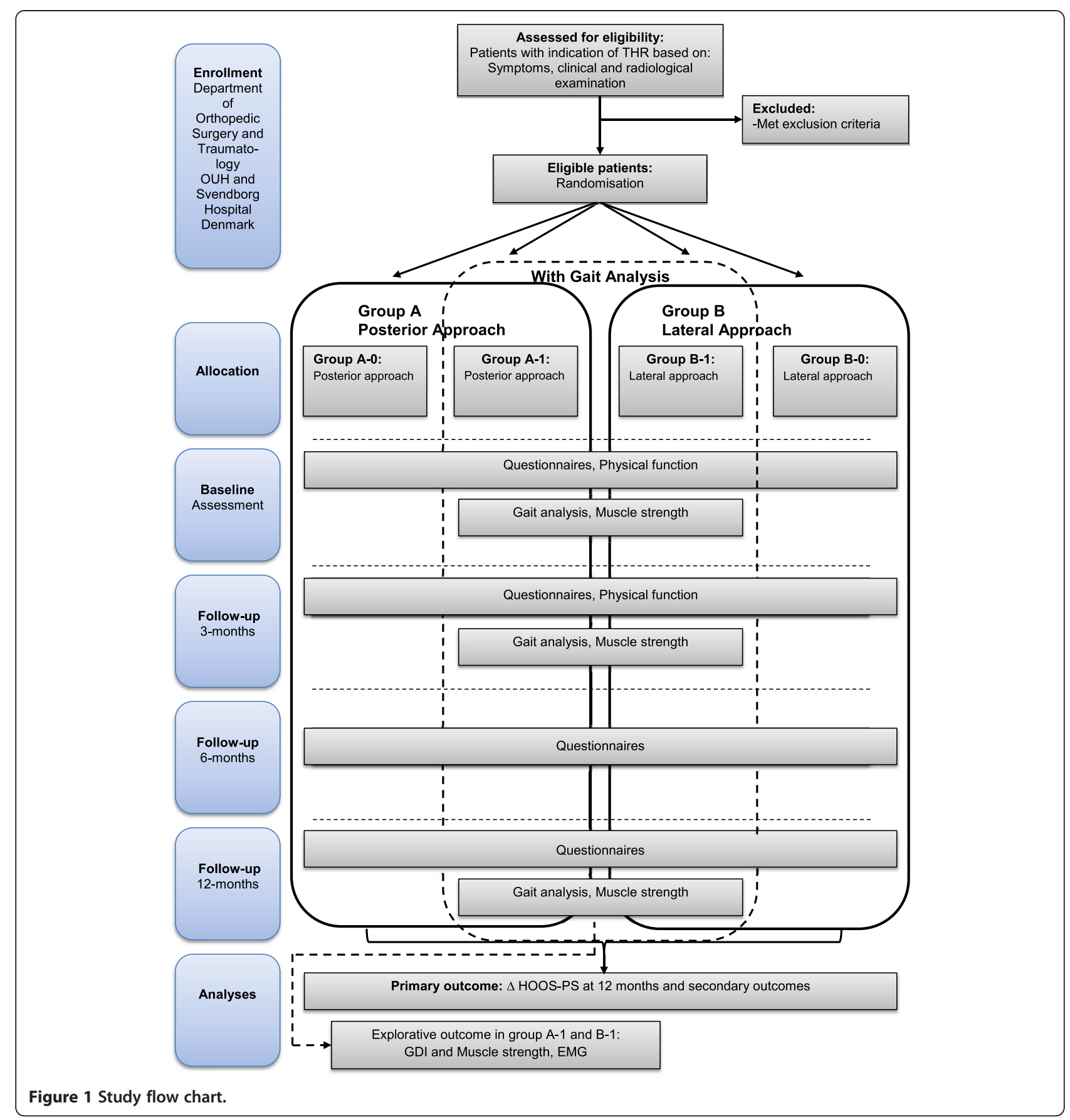

\section{Statistical analysis}

Baseline characteristic of study participants, will be presented with mean and SD, for all relevant outcomes, according to CONSORT item 15 [19]. The primary statistical analysis will be performed between the two groups on mean change in HOOS-PS from baseline to twelve months follow-up. To evaluate the treatment effect (mean difference between the groups at three, six and twelve months), we will employ a random effect mixed linear model analysis (repeated measures) with point estimates [73]. This model includes the interaction between treatment and elapsed time, adjusted for baseline values and assuming that data is missing completely at random (MCAR) [73]. Model assumptions will be checked by residual plots. The secondary statistical analysis will include the same approach as described above for all the secondary outcomes. We will present the results with mean and standard deviation for both baseline and follow-up measurements in the two groups. Furthermore we will present the adjusted difference in mean between 
the groups at 12 months including 95\% CI. Finally, the effect size using Cohen's $d$ effect size with indexes for small, medium and large effect as proposed by Cohen, 1992 will be reported [74]. The intention-to-treat (ITT) principle will be applied [75]. Subsequent per-protocol analysis may be needed in case a substantial number of data is lost during follow-up. All data will be checked for Gaussian distribution, and parametric statistics will be used were appropriate. An $\alpha$-level of 0.05 will be used, and data presented as means with $95 \mathrm{CI}$, unless otherwise stated. Finally, the number needed to treat (NNT) for a positive effect of treatment ( $>10$ points on HOOS-PS) will be analysed. All statistical analyses will be blinded and performed using Stata 13 software (StataCorp, Texas, USA).

\section{Ethics}

The trial complies with the Declaration of Helsinki. It is approved by the Danish Data Protection Agency and The Danish Regional Committee on Biomedical Research Ethics (Southern Denmark), Project-ID S-20120009. A written and orally informed consent will be collected prior to inclusion of all participants.

\section{Discussion}

The choice of surgical approach and the influence on patient-reported outcome, physical function, hip muscle function and gait are often debated. Especially, the lateral approach and its impact on the hip abductor muscles are a concern that may be associated with decreased PROM, reduced physical function and increased limping. Interest in reducing the surgical induced muscle damaged is also emphasised by a study showing that decreased lower limb muscle strength is associated with deterioration of ADL functions [76]. Despite this, only few studies have evaluated PROM, physical function and gait between the PA group and LA group.

\section{PROM}

The trial focuses on HOOS-PS as primary outcome after twelve months, along with a number of secondary outcomes, in order to evaluate the patients' perspective on physical function (HHS-limping), physical activity (UCLA), pain (HOOS-Pain, EQ-5D) and quality of life (HOOS-QoL, EQ-5D). All of these are valid measurements and recommended for use in RCTs [77].

To our knowledge, only three studies have investigated PROM when comparing PA with LA $[6,11,18]$. Of these only one study is a randomised controlled trial (RCT) and did not use PROM as primary outcome. Furthermore, the three studies do not agree in their conclusions of which approach is superior in terms of patient reported physical function and pain. The two non-randomised cohort studies are demonstrating a small, but significant, difference in favour of PA. Contrary, the RCT did not find any differences in WOMAC, which was evaluated as a secondary outcome. Also, two studies have compared the PA with LA using Harris Hip Score (HHS) [12,18]; however, HHS is not entirely patient-reported. The first study found that LA had a significant inferior outcome on total HHS after twelve months [12], whereas the other study found no differences after three months [18].

PROM is recommended by OMERACT (Outcome Measures in Rheumatologic Clinical Trials) as the core set of outcome measures in phase III clinical trials investigating OA treatment, including THR [77]. The core set outcome includes pain, physical function and patient global assessment. We have chosen the HOOS-PS as primary outcome for mainly three reasons. First, physical function has been defined by OMERACT as one out of three core set outcomes to evaluate. Physical function is well reflected in HOOS-PS, which includes 5 items. Second, we find it relevant to use HOOS-PS as primary outcome, because it reflects the possible negative influence of LA due to the surgical damage on the hip abductor muscles, which could lead to reduced physical function for these patients. Third, HOOS is in a recent review recommended for evaluation of THR patients, because it was found to be the best validated and a reliable PROM when compared with other commonly used PROMs (WOMAC, OHS, modified HHS etc.) [22].

Pain is also a core set outcome in the OMERACT guidelines [77]. Measurements of pain are represented in both the secondary and the explorative outcomes, by HOOS-pain, NRS and actual intake of painkillers. Lastly, a global patient assessment score is the third core set outcome in the OMERACT guidelines, and it will be measured by the patients' perception of their overall health with the second part of EQ-5D. The use of a specific question regarding limping from HHS will provide important information on the gait quality perceived by the patients and will be further explored using GDI.

\section{Physical function}

The surgical induced damaged to the abductor muscles (muscle gluteus medius and gluteus minimus) may contribute to a decrease of the physical function in the LA group $[6,7,17]$. Evaluating the overall early physical function and the gait performance will contribute with data that explore this hypothesis further. Also, the physical function test has shown greater responsiveness during the early stage after surgery than PROM [42], and it has been shown that the physical function test may capture a different construct of the patients function than patient-reported physical function. Patient-reported physical function has shown closer relation to the patients' perception of pain [42]. For these reasons, the trial is designed with early measurement of the patients' physical function, using a battery of valid and reliable tests. 


\section{Gait and muscle strength}

Several trials have found reduced maximal voluntary muscle strength (MVC) of the hip abductor muscles in the affected side amongst the THR population [58,78-80]. However, only three non-randomised studies have investigated MVC of the abductor muscles comparing PA with LA. Gore et al. [15] found reduced abductor muscle strength in the LA group, while equal muscle strength between the two approaches was reported by Downing et al. [81] and Kiyama et al. [78]. Thus, no firm conclusion can be draw from the existing literature. Previously, hip abductor muscle weakness during gait, evaluated with 3D-gait analysis, has also been reported in the general THR population $[56,60,79,82]$. However, only one study investigating the quality of gait using 3D-gait analysis has compared LA with PA. This study concluded that all the LA-patients demonstrated an abnormal gait pattern, while $30 \%$ of the PA-patients had a normal gait pattern six month after surgery [17]. It was suggested that the observed abnormal gait pattern in the LA-group was a compensatory mechanism potential caused by hip abductor weakness, even though muscle strength was not assessed [17]. A recent review investigating gait quality of the THR-population compared with healthy controls found impairment in several outcomes, including gait speed and hip abductor muscle function in the THR group [83]. Because only few studies until now have been conducted, Ewen et al. emphasise the importance of investigating the potential differences between surgical approaches in the future [83]. In summary, the quality and quantity of information extracted from studies comparing LA with PA performed to date are insufficient to draw any firm conclusions about physical function, muscle strength and gait.

\section{Study design}

The internal validity of the trial is good, due to same recruitment procedure, same in/exclusion criteria, same team taking care of the patients, and the patients experience the same amount of extra attention regarding the additional outpatient visits related to testing. The age limits and the list of exclusion criteria can affect the external validity and generalizability. However; patients under the age of 45 are likely to suffer from secondary OA and in our department all patients over the age of 70 receive a standard treatment with a cemented prosthetic concept. Thus, to ensure high internal validity by avoiding subgroup analysis due to different prosthetic concepts, we decided only to include patients aged 45-70. The exclusion criteria facilitate investigation of the potential differences between the two approaches due to the impact on different soft tissue structures. Thus, the exclusion criteria enable the investigation of the isolated effect of the two approaches, without interference of any other health conditions, which is the primary aim of this trial. We have chosen the primary end-point at twelve months after operation, as we expect a stable rehabilitations level at this point [42]. The intermediate assessments at three and six months will provide information about potential differences in the progression of rehabilitation between the two groups.

We have chosen block randomisation for two reasons: 1) because logistic challenges exist regarding booking of patients to the surgical theatre combined with a surgeon from either the LA or PA team. In this way, we ensure that patients do not wait an unnecessarily long time for operation, after they are included. 2) The higher chance (66\%) of being allocated to gait analysis in the first three blocks will enable us to verify the sample size for GDI. If needed, an adjusted allocation to gait analysis will be possible in the following block.

We have chosen to randomise the patients to a specific approach that automatically includes a specific team of surgeons with specialised skills in that particular approach, thus avoiding bias due to a learning curve. To avoid the risk of comparing surgeon skills and/or preference rather than the surgical procedure, a team of three experienced surgeons has been included in each group.

The randomisation code will be revealed to the investigators after the data analysis has been finalised. The primary outcome and supplementary PROM outcome will be blinded to the data collectors and data assessor (SR) throughout the trial, since all questionnaires will be answered at the patient's home. The data collectors are also blinded to intervention when collecting data regarding the physical test. However, some difficulties regarding blinding must be mentioned. For practical reasons, it is not possible to maintain the blinding to intervention for the data collector (DBN) regarding the 3D gait analysis at three and twelve months' follow-up, when placing the EMG electrodes in close relation to the scar at the hip. However, this will only influence a subgroup of the trial population and only the collection of explorative outcomes. Also, we will blind the patients by not telling them the given intervention, and prior to intervention we will explain to them why it is important that they are retained from this information. Nevertheless, there is a risk that the patient will guess the intervention from the name of the surgeon.

\section{Conclusion}

The present trial will provide evidence for the choice of approach in the future, with focus on the patients' perspective. The results of the trial will be submitted to peer-reviewed journals for publication, irrespective of the outcome, in accordance with the CONSORT guidelines for reporting of clinical trials and registration in ClinicalTrails.gov [84]. 


\section{Abbreviations}

OA: Osteoarthritis; THR: Total hip replacement; PA: Posterior approach; LA: Lateral approach; PROM: Patient-reported outcome measures; RCT: Randomised controlled trial; 30s CST: 30 second chair-stand-test; CE-angle: Center-edge-angle; NRS: Numeric Ranking Scale; HOOS: Hip Disability and Osteoarthritis Outcome Score; EQ-5D: EuroQol;

UCLA: University of California Los Angeles activity score; HHS: Harris Hip Score; 20WT: 20 meter walk test; TUG: Timed Up and Go; 30s knee bend: 30 sec repeated unilateral knee bending test; ROM: Range of motion; GDI: Gait Deviation Index; MVC: Isometric maximal voluntary muscle contraction; WOMAC: Western Ontario and McMaster Universities Osteoarthritis Index; OHS: Oxford Hip Score.

\section{Competing interests}

The authors declare that they have no conflicts of interests. The patients attending this trial will be involved in additional clinical examinations, beside the standard program. The patients get a refund on the additional transportations cost they experience. They do not receive any payment to participate.

\section{Authors' contributions}

All authors participated in the design of the trial. SR serves as the principal investigator for the trial and is responsible for drafting this paper. $C J, A H L, L B$, SO have commented and revised drafts. All authors have read and approved the final manuscript.

\section{Acknowledgement}

We would like to acknowledge Jens Lauritzen (JL) for his advice on the sample size, randomisation sequence and statistical analysis sections. We would also like to acknowledge all the surgeons involved in the inclusion and/or operation of patients (Ole Ovesen, Hans Christian Kærlev, Morten Foged Bøgehøj, Dina Pedersen, Ole Jakobsen, Sead Hasific og Ahsan Al Maleh, René Kirchhoff- Jensen og Jorg Dominik Adam). The trial is funded by the Bjarne Jensens Foundation, Bevica Foundation, The Danish Rheumatism Association, The Region of Zealand, Research Council at Odense University hospital, The Region of Southern Denmark, and the University of Southern Denmark.

Received: 12 August 2013 Accepted: 9 October 2014

Published: 27 October 2014

\section{References}

1. Ethgen O, Bruyere O, Richy F, Dardennes C, Reginster JY: Health-related quality of life in total hip and total knee arthroplasty. A qualitative and systematic review of the literature. J Bone Joint Surg Am 2004, 86-A:963-974

2. Nilsdotter AK, Petersson IF, Roos EM, Lohmander LS: Predictors of patient relevant outcome after total hip replacement for osteoarthritis: a prospective study. Ann Rheum Dis 2003, 62:923-930.

3. Steiner C, Andrews R, Barrett M, Weiss A: HCUP Projections Report \# 2012-03. In HCUP Projections: Mobility/Orthopedic Procedures 2011 to 2012. U.S. Agency for Healthcare Research and Quality; 2012. ONLINE September 20, 2012. [http://www.hcup-us.ahrq.gov/reports/projections/2012-03.pdf]

4. Annual Report 2009, Danish Hip Arthroplasty Registry. March 2010. 2009 [http://www.dhr.dk/Ny\%20mappe/rapporter/DHR\%20Aarsrapport_2009\% 20t_web.pdf]

5. Havelin LI, Fenstad AM, Salomonsson R, Mehnert F, Furnes O, Overgaard S, Pedersen AB, Herberts P, Karrholm J, Garellick G: The Nordic Arthroplasty Register Association: a unique collaboration between 3 national hip arthroplasty registries with 280,201 THRs. Acta Orthop 2009, 80:393-401.

6. Palan J, Beard DJ, Murray DW, Andrew JG, Nolan J: Which approach for total hip arthroplasty: anterolateral or posterior? Clin Orthop Relat Res 2009, 467:473-477.

7. Jolles BM, Bogoch ER: Posterior versus lateral surgical approach for total hip arthroplasty in adults with osteoarthritis. Cochrane Database Syst Rev 2004. Issue 3. Art. No.: CD003828.

8. Arthursson AJ, Furnes O, Espehaug B, Havelin LI, Soreide JA: Prosthesis survival after total hip arthroplasty-does surgical approach matter? Analysis of 19,304 Charnley and 6,002 Exeter primary total hip arthroplasties reported to the Norwegian Arthroplasty Register. Acta Orthop 2007, 78:719-729.
9. Hailer NP, Weiss RJ, Stark A, Karrholm J: The risk of revision due to dislocation after total hip arthroplasty depends on surgical approach, femoral head size, sex, and primary diagnosis. Acta Orthop 2012, 83:442-448.

10. Pellicci PM, Bostrom M, Poss R: Posterior approach to total hip replacement using enhanced posterior soft tissue repair. Clin Orthop Relat Res 1998, 355:224-228.

11. Smith AJ, Wylde V, Berstock JR, Maclean AD, Blom AW: Surgical approach and patient-reported outcomes after total hip replacement. Hip Int 2012, 22(4):355-361.

12. Edmunds $C T$, Boscainos PJ: Effect of surgical approach for total hip replacement on hip function using Harris Hip scores and Trendelenburg's test. A retrospective analysis. Surgeon 2011, 9:124-129.

13. Masonis JL, Bourne RB: Surgical approach, abductor function, and total hip arthroplasty dislocation. Clin Orthop Relat Res 2002, 405:46-53.

14. Mulliken $\mathrm{BD}$, Rorabeck $\mathrm{CH}$, Bourne RB, Nayak N: A modified direct lateral approach in total hip arthroplasty: a comprehensive review. $J$ Arthroplasty 1998, 13:737-747.

15. Gore DR, Murray MP, Sepic SB, Gardner GM: Anterolateral compared to posterior approach in total hip arthroplasty: differences in component positioning, hip strength, and hip motion. Clin Orthop Relat Res 1982 165:180-187.

16. Baker AS, Bitounis VC: Abductor function after total hip replacement. An electromyographic and clinical review. J Bone Joint Surg Br 1989, 71:47-50.

17. Madsen MS, Ritter MA, Morris HH, Meding JB, Berend ME, Faris PM, Vardaxis VG: The effect of total hip arthroplasty surgical approach on gait. J Orthop Res 2004, 22:44-50.

18. Witzleb WC, Stephan L, Krummenauer F, Neuke A, Gunther KP: Short-term outcome after posterior versus lateral surgical approach for total hip arthroplasty - A randomized clinical trial. Eur J Med Res 2009, 14:256-263.

19. Moher D, Hopewell S, Schulz KF, Montori V, Gotzsche PC, Devereaux PJ, Elbourne D, Egger M, Altman DG: CONSORT 2010 explanation and elaboration: updated guidelines for reporting parallel group randomised trials. BMJ 2010, 340:c869.

20. Hoppenfeld S, de Boer P, Buckley R: The Hip. In Surgical Exposures in Orthopaedics the Anatomic Approach. 4th edition. Philadelphia: Lippincott Williams \& Wilkins; 2009:403-462.

21. Davis AM, Perruccio AV, Canizares M, Tennant A, Hawker GA, Conaghan PG, Roos EM, Jordan JM, Maillefert JF, Dougados M, Lohmander LS: The development of a short measure of physical function for hip OA HOOS-Physical Function Shortform (HOOS-PS): an OARSI/OMERACT initiative. Osteoarthritis Cartilage 2008, 16:551-559.

22. Thorborg K, Roos EM, Bartels EM, Petersen J, Holmich P: Validity, reliability and responsiveness of patient-reported outcome questionnaires when assessing hip and groin disability: a systematic review. Br J Sports Med 2010, 44:1186-1196.

23. Nilsdotter AK, Lohmander LS, Klassbo M, Roos EM: Hip disability and osteoarthritis outcome score (HOOS)-validity and responsiveness in total hip replacement. BMC Musculoskelet Disord 2003, 4:10.

24. Davis AM, Perruccio AV, Canizares M, Hawker GA, Roos EM, Maillefert JF, Lohmander LS: Comparative, validity and responsiveness of the HOOS-PS and KOOS-PS to the WOMAC physical function subscale in total joint replacement for osteoarthritis. Osteoarthritis Cartilage 2009, 17:843-847.

25. de Groot IB, Reijman M, Terwee CB, Bierma-Zeinstra SM, Favejee M, Roos EM, Verhaar JA: Validation of the Dutch version of the Hip disability and Osteoarthritis Outcome Score. Osteoarthritis Cartilage 2007, 15:104-109.

26. Beyer N, Thorborg K, Vinther A: Translation and Cross-Cultural Adaptation of the Danish Version of the Hip Dysfunction and Osteoarthritis Outcome Score 2.0 (HOOS 2.0). 2008 [http://koos.nu/index.html]

27. Brooks R: EuroQol: the current state of play. Health Policy 1996, 37:53-72.

28. Fransen $\mathrm{M}$, Edmonds J: Reliability and validity of the EuroQol in patients with osteoarthritis of the knee. Rheumatology (Oxford) 1999, 38:807-813.

29. Ashby E, Grocott MP, Haddad FS: Outcome measures for orthopaedic interventions on the hip. J Bone Joint Surg Br 2008, 90:545-549.

30. van Agt HM, Essink-Bot ML, Krabbe PF, Bonsel GJ: Test-retest reliability of health state valuations collected with the EuroQol questionnaire. Soc Sci Med 1994, 39:1537-1544.

31. Harrison MJ, Davies LM, Bansback NJ, Ingram M, Anis AH, Symmons DP: The validity and responsiveness of generic utility measures in rheumatoid arthritis: a review. J Rheumatol 2008, 35:592-602. 
32. Tidermark J, Bergstrom G, Svensson O, Tornkvist H, Ponzer S: Responsiveness of the EuroQol (EQ 5-D) and the SF-36 in elderly patients with displaced femoral neck fractures. Qual Life Res 2003, 12:1069-1079.

33. Paulsen A, Pedersen AB, Overgaard S, Roos EM: Feasibility of 4 patient-reported outcome measures in a registry setting. Acta Orthop 2012, 83:321-327.

34. Translation Process-EuroQol. [http://www.euroqol.org/eq-5d-products/ translation-process.html]

35. Terwee CB, Bouwmeester W, van Elsland SL, de Vet HC, Dekker J: Instruments to assess physical activity in patients with osteoarthritis of the hip or knee: a systematic review of measurement properties. Osteoarthritis Cartilage 2011, 19:620-633.

36. Naal FD, Impellizzeri FM, Leunig M: Which is the best activity rating scale for patients undergoing total joint arthroplasty? Clin Orthop Relat Res 2009, 467:958-965.

37. Beaule PE, Dorey FJ, Hoke R, Le DM, Amstutz HC: The value of patient activity level in the outcome of total hip arthroplasty. J Arthroplasty 2006, 21:547-552.

38. Soderman P, Malchau H: Is the Harris hip score system useful to study the outcome of total hip replacement? Clin Orthop Relat Res 2001, 384:189-197.

39. Villadsen A, Roos EM, Overgaard S, Holsgaard-Larsen A: Agreement and reliability of functional performance and muscle power in patients with advanced osteoarthritis of the hip or knee. Am J Phys Med Rehabil 2012, 91:401-410

40. Podsiadlo D, Richardson S: The timed "Up \& Go": a test of basic functional mobility for frail elderly persons. J Am Geriatr Soc 1991, 39:142-148.

41. Yeung TS, Wessel J, Stratford PW, MacDermid JC: The timed up and go test for use on an inpatient orthopaedic rehabilitation ward. J Orthop Sports Phys Ther 2008, 38:410-417.

42. Mizner RL, Petterson SC, Clements KE, Zeni JA Jr, Irrgang JJ, Snyder-Mackler $L$ : Measuring functional improvement after total knee arthroplasty requires both performance-based and patient-report assessments: a longitudinal analysis of outcomes. J Arthroplasty 2011, 26:728-737.

43. Kennedy DM, Stratford PW, Wessel J, Gollish JD, Penney D: Assessing stability and change of four performance measures: a longitudinal study evaluating outcome following total hip and knee arthroplasty. $B M C$ Musculoskelet Disord 2005, 6:3.

44. Wright AA, Cook CE, Baxter GD, Dockerty JD, Abbott JH: A comparison of 3 methodological approaches to defining major clinically important improvement of 4 performance measures in patients with hip osteoarthritis. J Orthop Sports Phys Ther 2011, 41:319-327.

45. Dobson F, Hinman RS, Hall M, Terwee CB, Roos EM, Bennell KL: Measurement properties of performance-based measures to assess physical function in hip and knee osteoarthritis: a systematic review. Osteoarthritis Cartilage 2012, 20:1548-1562.

46. Jones CJ, Rikli RE, Beam WC: A 30-s chair-stand test as a measure of lower body strength in community-residing older adults. Res Q Exerc Sport 1999, 70:113-119.

47. Gill S, McBurney H: Reliability of performance-based measures in people awaiting joint replacement surgery of the hip or knee. Physiother Res Int 2008, 13:141-152.

48. Bremander $A B, D a h l L L$, Roos EM: Validity and reliability of functional performance tests in meniscectomized patients with or without knee osteoarthritis. Scand J Med Sci Sports 2007, 17:120-127.

49. Hardcastle P, Nade S: The significance of the Trendelenburg test. J Bone Joint Surg Br 1985, 67:741-746.

50. Kendall KD, Patel C, Wiley JP, Pohl MB, Emery CA, Ferber R: Steps towards the validation of the Trendelenburg test: the effect of experimentally reduced hip abductor muscle function on frontal plane mechanics. Clin $J$ Sport Med 2012

51. Kendall KD, Schmidt C, Ferber R: The relationship between hip-abductor strength and the magnitude of pelvic drop in patients with low back pain. J Sport Rehabil 2010, 19:422-435.

52. Bergmann TF, Peterson DH, Lawrence DJ: Extraspinal Techniques. In Chiropractic Techniques. New York: Churchill Livingstone Inc; 1993.

53. Poulsen E, Christensen HW, Penny JO, Overgaard S, Vach W, Hartvigsen J: Reproducibility of range of motion and muscle strength measurements in patients with hip osteoarthritis - an inter-rater study. BMC Musculoskelet Disord 2012, 13:242

54. Schwartz MH, Rozumalski A: The Gait Deviation Index: a new comprehensive index of gait pathology. Gait Posture 2008, 28:351-357.
55. Pospischill M, Kranzl A, Attwenger B, Knahr K: Minimally invasive compared with traditional transgluteal approach for total hip arthroplasty: a comparative gait analysis. J Bone Joint Surg Am 2010, 92:328-337.

56. Foucher $\mathrm{KC}$, Hurwitz DE, Wimmer MA: Preoperative gait adaptations persist one year after surgery in clinically well-functioning total hip replacement patients. J Biomech 2007, 40:3432-3437.

57. Molloy M, McDowell BC, Kerr C, Cosgrove AP: Further evidence of validity of the Gait Deviation Index. Gait Posture 2010, 31:479-482.

58. Jensen C, Aagaard P, Overgaard S: Recovery in mechanical muscle strength following resurfacing vs standard total hip arthroplasty - a randomised clinical trial. Osteoarthritis Cartilage 2011, 19:1108-1116.

59. SENIAM EMG Guidelines. [http://www.seniam.org]

60. Perron M, Malouin F, Moffet H, McFadyen BJ: Three-dimensional gait analysis in women with a total hip arthroplasty. Clin Biomech (Bristol, Avon) 2000, 15:504-515.

61. Burden AM, Trew M, Baltzopoulos V: Normalisation of gait EMGs: a re-examination. J Electromyogr Kinesiol 2003, 13:519-532.

62. Burden A: How should we normalize electromyograms obtained from healthy participants? What we have learned from over 25 years of research. J Electromyogr Kinesiol 2010, 20:1023-1035.

63. Bergh I, Sjostrom B, Oden A, Steen B: An application of pain rating scales in geriatric patients. Aging (Milano) 2000, 12:380-387.

64. Zhang W, Moskowitz RW, Nuki G, Abramson S, Altman RD, Arden N, Bierma-Zeinstra S, Brandt KD, Croft P, Doherty M, Dougados M, Hochberg M, Hunter DJ, Kwoh K, Lohmander LS, Tugwell P: OARSI recommendations for the management of hip and knee osteoarthritis, Part II: OARSI evidence-based, expert consensus guidelines. Osteoarthritis Cartilage 2008, 16:137-162.

65. Paulsen A, Overgaard S, Lauritsen JM: Quality of data entry using single entry, double entry and automated forms processing-an example based on a study of patient-reported outcomes. PLoS One 2012, 7:e35087.

66. Ehrich EW, Davies GM, Watson DJ, Bolognese JA, Seidenberg BC, Bellamy N: Minimal perceptible clinical improvement with the Western Ontario and McMaster Universities osteoarthritis index questionnaire and global assessments in patients with osteoarthritis. J Rheumatol 2000, 27:2635-2641.

67. Roos EM, Lohmander LS: The Knee injury and Osteoarthritis Outcome Score (KOOS): from joint injury to osteoarthritis. Health Qual Life Outcomes 2003, 1:64

68. Terwee CB, Roorda LD, Knol DL, De Boer MR, De Vet HC: Linking measurement error to minimal important change of patient-reported outcomes. J Clin Epidemiol 2009, 62:1062-1067.

69. KOOS FAQs. [http://koos.nu/index.html]

70. Frison L, Pocock SJ: Repeated measures in clinical trials: analysis using mean summary statistics and its implications for design. Stat Med 1992, 11:1685-1704

71. Cimolin V, Galli M, Vimercati SL, Albertini G: Use of the Gait Deviation Index for the assessment of gastrocnemius fascia lengthening in children with Cerebral Palsy. Res Dev Disabil 2011, 32:377-381.

72. Rosenlund S, Nielsen DB, Overgaard S, Jensen C, Holsgaard-Larsen A: Is gait velocity and gait quality associated with hip muscle strength in hip osteoarthritis patients scheduled for total hip arthroplasty? Osteoarthritis Cartilage 2014, 22:S116-S117.

73. Rabe-Hesketh S, Skrondal A: In Multilevel and Longitudinal Modeling using Stata. 2nd edition. Edited by Newton HJ. Texas: Stata Press; 2008.

74. Cohen J: A power primer. Psychol Bull 1992, 112:155-159.

75. Sainani KL: Making sense of intention-to-treat. PMR 2010, 2:209-213.

76. Rantanen T, Avlund K, Suominen H, Schroll M, Frandin K, Pertti E: Muscle strength as a predictor of onset of $A D L$ dependence in people aged 75 years. Aging Clin Exp Res 2002, 14:10-15.

77. Bellamy N, Kirwan J, Boers M, Brooks P, Strand V, Tugwell P, Altman R, Brandt K, Dougados M, Lequesne M: Recommendations for a core set of outcome measures for future phase III clinical trials in knee, hip, and hand osteoarthritis. Consensus development at OMERACT III. J Rheumatol 1997, 24:799-802.

78. Kiyama T, Naito M, Shinoda T, Maeyama A: Hip abductor strengths after total hip arthroplasty via the lateral and posterolateral approaches. J Arthroplasty 2010, 25:76-80

79. Klausmeier V, Lugade V, Jewett BA, Collis DK, Chou LS: Is there faster recovery with an anterior or anterolateral THA? A pilot study. Clin Orthop Relat Res 2010, 468:533-541. 
80. Rasch A, Dalen N, Berg HE: Muscle strength, gait, and balance in 20 patients with hip osteoarthritis followed for 2 years after THA. Acta Orthop 2010, 81:183-188.

81. Downing ND, Clark DI, Hutchinson JW, Colclough K, Howard PW: Hip abductor strength following total hip arthroplasty: a prospective comparison of the posterior and lateral approach in 100 patients. Acta Orthop Scand 2001, 72:215-220.

82. Beaulieu ML, Lamontagne M, Beaule PE: Lower limb biomechanics during gait do not return to normal following total hip arthroplasty. Gait Posture 2010, 32:269-273.

83. Ewen AM, Stewart S, St Clair GA, Kashyap SN, Caplan N: Post-operative gait analysis in total hip replacement patients-a review of current literature and meta-analysis. Gait Posture 2012, 36:1-6.

84. Boutron I, Moher D, Altman DG, Schulz KF, Ravaud P: Extending the CONSORT statement to randomized trials of nonpharmacologic treatment: explanation and elaboration. Ann Intern Med 2008, 148:295-309.

doi:10.1186/1471-2474-15-354

Cite this article as: Rosenlund et al:: The effect of posterior and lateral approach on patient-reported outcome measures and physical function in patients with osteoarthritis, undergoing total hip replacement: a randomised controlled trial protocol. BMC Musculoskeletal Disorders 2014 15:354.

\section{Submit your next manuscript to BioMed Central and take full advantage of:}

- Convenient online submission

- Thorough peer review

- No space constraints or color figure charges

- Immediate publication on acceptance

- Inclusion in PubMed, CAS, Scopus and Google Scholar

- Research which is freely available for redistribution 